\title{
Expression patterns of SLIT/ROBO mRNAs reveal a characteristic feature in the entorhinal-hippocampal area of macaque monkeys
}

Tetsuya Sasaki $i^{1,2,3^{*}} \mathbb{D}$, Yusuke Komatsu ${ }^{1,4}$ and Tetsuo Yamamori ${ }^{1,3^{*}}$

\begin{abstract}
Objective: SLITs are secreted glycoproteins that bind to Roundabouts (ROBOs) which are a family member of transmembrane receptors. SLIT signaling has well-conserved roles in mediating axon repulsion in a developing nervous system. We previously reported that SLIT1 mRNA is enriched in middle layers of the prefrontal cortex of macaque monkeys in a developmentally regulated manner. Other SLIT (SLIT2 and SLIT3) mRNAs showed preferential expressions in the prefrontal cortex with a distinct laminar pattern. To obtain further clues to the role of SLIT signaling in the organization of the primate brain, we performed ISH analysis of SLIT and ROBO mRNAs using adult macaque brain tissues.

Results: In this study, we examined the expression patterns of SLITs and ROBOs (ROBO1 and ROBO2) in other brain regions, and found intense and characteristic expression patterns of these genes in the entorhinal-hippocampal area. In situ hybridization analysis revealed that SLIT1 and SLIT2 mRNAs showed marked complementary distribution in the entorhinal cortex. SLIT and ROBO mRNAs were widely expressed in the hippocampus with modest regional preference. These findings suggest that each SLIT gene has a specialized role that is particularly important for prefrontal as well as hippocampal connectivity in the primate cortex.
\end{abstract}

Keywords: Axon guidance, Cerebral cortex, Entorhinal cortex, Hippocampus, In situ hybridization, Primates

\section{Introduction}

SLIT is a repellent guidance molecule, which is well conserved in various species $[1,2]$. The repellent effect of SLIT is mediated by the receptor, Roundabout (ROBO) [3]. To date, three Slit genes, Slit1-3 [4] and four Robo genes (Robo1-4) have been identified in vertebrate genome [3, 5-7]. In mammals, SLIT/ROBO signaling is

\footnotetext{
*Correspondence: tsasaki@md.tsukuba.ac.jp; tetsuo.yamamori@riken.ac.jp ${ }^{2}$ Present Address: Department of Anatomy and Neuroscience, Faculty of Medicine, University of Tsukuba, 1-1-1 Tennodai, Tsukuba, Ibaraki 305-8577, Japan

${ }^{5}$ Laboratory for Molecular Analysis of Higher Brain Function, RIKEN Center for Brain Science, Wako, Saitama 351-0198, Japan

Full list of author information is available at the end of the article
}

reported to have essential roles in the development of the nervous system including midline crossing, as observed in Drosophila $[8,9]$ and formation of major axonal tracts [10-13]. Furthermore, recent studies demonstrate expanded functional repertories of SLITs and ROBOs, such as neurogenesis, cell proliferation/migration, angiogenesis, oncogenesis, and involvement in several diseases [2, 14-17].

We have investigated the molecular basis of differences in the architecture across neocortical areas and identified three genes, SLIT1 [18], RBP4 [19], and PNMA5 [20] that are highly expressed in the higher-order association areas of macaque monkeys [21]. Among them, SLIT1 mRNA in particular is preferentially expressed in the prefrontal 
cortex compared with other association areas. Our detailed in situ hybridization (ISH) analysis demonstrates that SLIT1 mRNA is mainly distributed in the middle layers of most cortical areas, highest in the prefrontal cortex but lowest in the primary sensory areas. The prefrontalenriched pattern was established by reduced expressions, specific for areas and layers during postnatal development. The promoter region of SLIT1 gene is hypermethylated, and it is assumed that some regulatory elements (e.g., methyl-binding proteins) are involved in the area selective expression [22]. Other SLIT (SLIT2 and SLIT3) mRNAs showed modest preference in the prefrontal cortex, whereas $R O B O 1$ and $R O B O 2$ mRNAs were widely detected within the cerebral cortex. Since cortical neurons, particularly those in the prefrontal cortex, simultaneously express SLIT1 and ROBO mRNAs, SLIT1 could work in either an autocrine or paracrine manner in the postnatal primate cortex, which implies that it has other functions in addition to its role as guidance cues.

In this report, to obtain further clues to the role of SLIT signaling in the organization of the primate brain, we performed ISH analysis of SLIT and ROBO mRNAs using adult macaque brain tissues. We examined the expression patterns of these genes in detail in brain regions other than the prefrontal cortex. We found unique expression patterns of these genes in the entorhinal-hippocampal area.

\section{Main text \\ Materials and methods \\ Experimental animals}

For ISH experiments, brains from five macaques (Macaca fuscata, juvenile to young adults, 2.6, 4.0, 5.2, 5.6, and 5.8 years old) were used. The animals were anesthetized and the brain fixed as described previously [18]. Sections from the blocks that included the entorhinal cortex and hippocampus were sliced at $35 \mu \mathrm{m}$ thickness (Additional file 1: Figure S1).

\section{In situ hybridization}

The cDNA fragments were obtained by RT-PCR using the primers listed in Table 1 , and subcloned into the pBlueScriptII vector. The concentrations of all the riboprobes used in this study were adjusted to $0.1 \mu \mathrm{g} / \mu \mathrm{l}$ and

Table 1 ISH probes used in this study

\begin{tabular}{|c|c|c|c|c|c|}
\hline Gene name & Probe name & Species & Accession No. & PCR primer set & Length \\
\hline \multirow[t]{8}{*}{ SLIT1 } & Slit1-1 & macaque & NM_003061 & cttccaggacctgcagaacc & 552 \\
\hline & & & & cccgtcttcgatctcggaca & \\
\hline & Slit1-2 & macaque & NM_003061 & aagtttgaatgccaaggtcc & 448 \\
\hline & & & & actgggectcgtgttgacat & \\
\hline & Slit1-3 & macaque & NM_003061 & cttgtgctctccggatctga & 822 \\
\hline & & & & gtacaggtttcggatgcaac & \\
\hline & Slit1-4 & macaque & NM_003061 & cctgtggcagatcctcaacg & 647 \\
\hline & & & & ccatcgctgcactcaaaggt & \\
\hline \multirow[t]{4}{*}{ SLIT2 } & Slit2-1 & macaque & NM_00478 & cccaggaatatcccccgcaa & 770 \\
\hline & & & & gagaccatcacagaaatacg & \\
\hline & Slit2-4 & macaque & NM_004787 & cagcccctgtgataattttg & 866 \\
\hline & & & & gtcctctgtgatgaagagga & \\
\hline \multirow[t]{2}{*}{ SLIT3 } & Slit3-3 & macaque & NM_003062 & ttgacctgagcaacaacagc & 838 \\
\hline & & & & ccctggacaaaggattcag & \\
\hline \multirow[t]{6}{*}{ ROBO1 } & Robol-1 & macaque & NM-022188 & ggagaggctgtgagccacaa & 942 \\
\hline & & & & tcctgtgaatcagactgtag & \\
\hline & Robo1-3 & macaque & NM-022188 & tggttagtttttgaagtgag & 877 \\
\hline & & & & acctacagtcgeccagctga & \\
\hline & Robo1-4 & macaque & NM-022188 & ctgatgctccctgagtcaac & 868 \\
\hline & & & & ggctacatttcaggacccct & \\
\hline \multirow[t]{4}{*}{$\mathrm{ROBO} 2$} & Robo2-1 & macaque & NM_002942 & aggaactatcttggtgaagc & 700 \\
\hline & & & & ggaaacccacagccagctgt & \\
\hline & Robo2-4 & macaque & NM_002942 & ccaggccaaggggataaaac & 673 \\
\hline & & & & gcctatcagtttgatatagc & \\
\hline
\end{tabular}


the riboprobes were stored at $-30{ }^{\circ} \mathrm{C}$. Single-color ISH was carried out essentially as previously described [18, 23 ]. We used more than two types of probe for each of SLIT1, SLIT2, ROBO1, and ROBO2, and confirmed that each probe exhibits the same pattern of signal distribution (data not shown). After the initial confirmation, multiple probes were mixed together to enhance ISH signals. We also confirmed that the sense probes detected no more signals above the background level. The layer positions of entorhinal cortex and the boundaries with hippocampal fields were determined on the basis of Cresyl violet staining of adjacent sections. Cresyl violet staining shows clear differences in cell size and packing density among the layers in the entorhinal cortex and the four CA fields in hippocampus [16, 24, 25].

\section{Image analysis}

Eight-bit gray scale color images were obtained using the digital color camera DP 70 (Olympus, Tokyo, Japan) attached to a BX-51 microscope (Olympus). The background image was subtracted using Image-Pro Plus image analysis software (Media Cybernetics, Silver Springs, MD). The laminar distribution patterns of different ISH signals (Fig. 1j, n) were analyzed as previously described [18]. Signals were extracted from the background component by converting the eight-bit gray-scale images into the binary images. The threshold used here was set to the standard deviation (SD) beyond the average intensity of each cortical section. Then, we calculated average values with respect to each row to obtain the line profile in regions of interest (ROIs: $100 \mu \mathrm{m}$ bin, which is the height of the cortex from layer I to WM) using Image Pro Plus.

\section{Results and discussion}

SLITs/ROBOs are reported to be involved in the formation of hippocampal connections during the rodent development [26]. We examined the SLIT and ROBO mRNA expression patterns in the hippocampus and its surrounding areas in the adult macaques (Additional file 1: Figure S1).

In and around the hippocampus, all the SLIT and $R O B O$ mRNAs were widely expressed (Fig. 1a-f). Each gene showed moderate regional preference. For example, the most intense signals of SLIT1 and ROBO1 mRNAs were observed in the granule cell layer of the dentate gyrus (DG, Fig. 1a, d), followed by the expression in the pyramidal cell layer of cornus ammmon (CA) 2-CA4. The expression levels of SLIT1 and ROBO1 mRNAs were rather low in CA1 and the subiculum. SLIT2 and ROBO2 mRNAs were also highly expressed in the DG and uniformly distributed in CA1-CA4 (Fig. 1b, e). SLIT3 mRNA

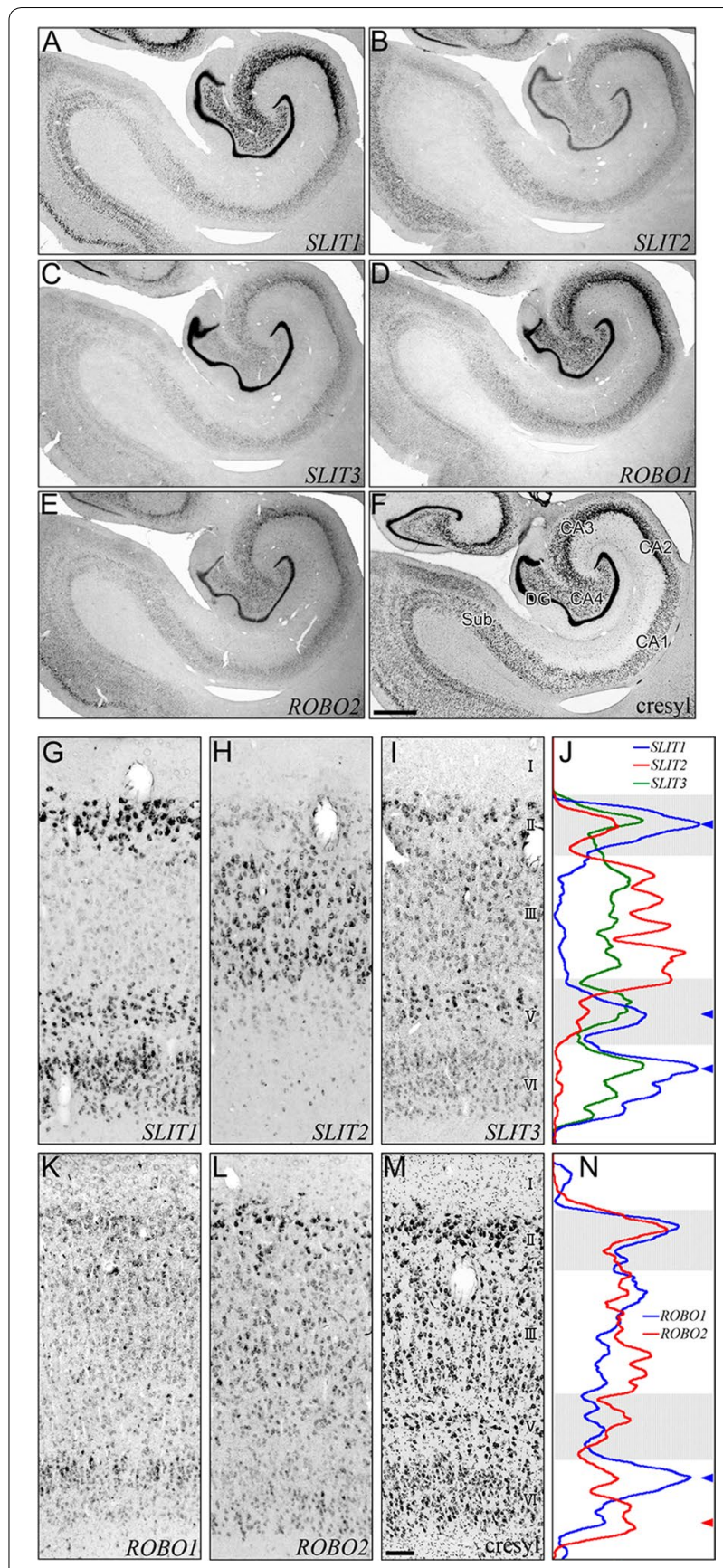

Fig. 1 Expression of SLIT and ROBO mRNAs in Hippocampus and Entorhinal Cortex. In situ hybridized sections for detection of SLIT1 (a), SLIT2 (b), SLIT3 (c), ROBO1 (d), and ROBO2 (e), and cresyl violet-stained section (f) of the hippocampus. DG: dentate gyrus; Sub: subiculum; ProS: prosubiculum; PrS: presubiculum. Scale bar $=1 \mathrm{~mm}$. Layer

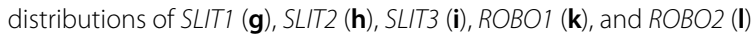
mRNAs in entorhinal cortex. Adjacent section for cresyl violet staining (m). Scale bar $=100 \mu \mathrm{m}$. The laminar profiles indicate the layer distributions of SLITS (j) and ROBOS (n). The density profiles of SLITI (blue), SLIT2 (red) and SLIT3 (green) were plotted along cortical depth (j). The density profiles of $R O B O 1$ (blue) and $R O B O 2$ (red) were plotted along cortical depth (n) 
was mainly expressed in the DG and relatively weak in other regions (Fig. 1c).

The entorhinal cortex is the interface between the hippocampus and the neocortex [27, 28]. It contains grid cells with their unique firing discharge pattern, and was reported as the key area for spatial representation in mammals [29, 30]. Abnormalities in the cells of layer II of the human entorhinal cortex have been implicated in the pathophysiology of schizophrenia and Alzheimer's disease [31, 32]. In the entorhinal cortex, Densitometric analysis indicated conspicuous laminar preference of SLIT and ROBO mRNAs. Most strikingly, SLIT1 and SLIT2 mRNAs showed complementary distribution (Fig. 1j). Intense signal of SLIT1 mRNA was observed in layers II, V, and VI (Fig. 1g and j, blue arrowheads) with only a low signal intensity of SLIT1 mRNA in layer III. Large cells that constitute the characteristic cell islands in layer II [16] showed a particularly high signal intensity of SLIT1 mRNA. On the other hand, SLIT2 mRNA was predominantly expressed in layer III (Fig. 1j), with only a weak expression in other layers (Fig. 1h). This complementarity is reminiscent of the pattern in area TE except that SLIT1 and SLIT2 mRNAs are expressed in different layers [18]. Owing to the different laminar preferences of these genes, we observed a sharp border between the entorhinal cortex and the perirhinal cortex (data not shown). SLIT3 mRNA was expressed similarly across layers in the entorhinal cortex, although intense signals were observed in the layer II and the upper part of layer V. $R O B O 1$ and $R O B O 2$ were also expressed widely across layers, which is similarly observed in other cortical areas. Interestingly, $\mathrm{ROBO} 1$ and $\mathrm{ROBO} 2 \mathrm{mRNAs}$ were abundant in the upper and lower parts of layer VI, as indicated by blue and red arrowheads in Fig. 1n, respectively, but the pattern not in neocortical areas. The differences in the laminar distribution among the SLITs in both the entorhinal cortex and neocortex might reflect different functions of these genes in the cerebral cortex of primates.

Although the roles of SLITs/ROBOs in the guidance of neurons during development may be expected, their functions in postnatal brains, where no major guidance events occur, remain unclear. A possible role of these molecules is in the control of regeneration in response to injury. All Slits are reported to be expressed in reactive astrocytes at the injury site portion of the rat brain [33]. Since $R O B O$ mRNAs are expressed in substantial cell populations in the cerebral cortex, SLITs secreted around the injury site may inhibit irrelevant axonal regeneration. Another plausible possibility is that the SLIT-ROBO system has a role in maintaining certain neuronal morphologies and circuits [34-37]. Lines of evidence suggest that axon guidance molecules are implicated as critical regulators in synaptogenesis and synaptic plasticity [37-39]. Numerous studies have shown the dynamics of neuronal processes in the postnatal cortex [40-42]. In this regard, we note that SLIT mRNAs were abundant in the brain regions where high neuronal integration and/or plasticity plays roles, such as the entorhinal cortex, dentate gyrus, and prefrontal cortex, where the other higherorder association area enriched genes, RBP4 and PNMA5 are also highly expressed $[19,20]$. These genes may subserve neural plasticity and cognitive function. Further research is needed to examine these possibilities.

\section{Limitations}

In this study, we found the characteristic expression pattern of the axon guidance molecule SLITs and its receptor ROBOs in the hippocampus and entorhinal cortex of adult macaque monkeys. We did not perform other methods such as qPCR or immunohistochemistry. Gene manipulation analysis including overexpression and suppression of gene expression will be needed to test our hypothesis that these groups of molecules are involved in structural plasticity of postnatal primate brains.

\section{Supplementary information}

Supplementary information accompanies this paper at https://doi. org/10.1186/s13104-020-05100-7.

Additional file 1: Figure S1. A coronal section of macaque brain containing hippocampus and entorhinal cortex for cresyl violet staining. Scale bar $=5 \mathrm{~mm}$. EC entorhinal cortex, HC hippocampus, LGN lateral geniculate nucleus, TEd dorsal inferotemporal cortex, TEv ventral inferotemporal cortex, sts superior temporal cortex, $P C$ perirhinal cortex, D dorsal, $V$ ventral, $L$ lateral, $M$ medial.

\section{Abbreviations}

CA: Cornus ammmon; DG: Dentate gyrus; ISH: In situ hybridization; ProS: Prosubiculum; PrS: Presubiculum; ROBO: Roundabouts; Sub: Subiculum.

\section{Acknowledgements}

We thank Kaoru Sawada and Sonoko Ohsawa of NIBB for their technical assistance. We also thank Dr. Fumiko Ono of the Corporation for Production and Research of Laboratory Primates, and Drs. Keiji Terao and Yuko Katakai of the Tsukuba Primate Research Center, National Institute of Infectious Diseases for supplying monkey tissues. We also thank the Open Facility, Research Facility Center for Science and Technology, University of Tsukuba.

\section{Authors' contribution}

TS and YK performed the experiments. TS and TY designed the study, and wrote the draft of the manuscript. All authors read and approved the final manuscript.

\section{Funding}

This work was mainly supported by a Scientific Research on Innovative Areas "Neural Diversity and Neocortical Organization (No. 22123009 to T.Y.)" from the Ministry of Education, Culture, Sports, Science, and Technology (MEXT), Japan. T.S. was supported by Grants-in-Aid for Young Scientists B (KAKENHI No. 17K16409), Grant-in-Aid for Scientific Research C (KAKENHI Nos. 19K08065), and Grant-in-Aid for Scientific Research on Innovative Areas "Multiscale Brain" (No. 19H05201) from MEXT Japan, the Takeda Science Foundation, the Naito Foundation, and by grants from the Brain Science Project of the Center of Novel Science Initiatives, National Institutes of Natural Sciences (BS271002, 
BS281009, and BS291005). This work was also supported by Spectrography and Bioimaging Facility, National Institute of Basic Biology, Core Research Facilities for technical support. Part of this study was carried out under the NIBB Cooperative Research Program (Nos. 19-509, 20-507) and the Cooperative Research Program of the Primate research Institute of Kyoto University (Nos. H30-B1, H31-B21).

\section{Availability of data and materials}

The datasets, which were used and/or analyzed in the current study, are available from the corresponding author on reasonable request.

\section{Ethics approval and consent to participate}

All the experiments described here were performed in compliance with the guidelines for animal experiments of the National Institutes of Natural Sciences, Japan and the National Institutes of Health, USA. All efforts were made to minimize animal suffering and the number of animals used.

\section{Consent for publication}

Not applicable.

\section{Competing interests}

The authors declare that they have no competing interests.

\section{Author details}

${ }^{1}$ Division of Brain Biology, National Institute for Basic Biology, 38 Nishigonaka Myodaiji, Okazaki 444-8585, Japan. ${ }^{2}$ Present Address: Department of Anatomy and Neuroscience, Faculty of Medicine, University of Tsukuba, 1-1-1 Tennodai, Tsukuba, Ibaraki 305-8577, Japan. ${ }^{3}$ Ph.D Program of Neurosciences, Graduate School of Comprehensive Human Sciences, University of Tsukuba, 1-1-1 Tennodai, Tsukuba, Ibaraki 305-8577, Japan. ${ }^{4}$ Laboratory of Veterinary Hygiene, Graduate School of Veterinary Medicine, Hokkaido University, Sapporo 060-0816, Japan. ${ }^{5}$ Laboratory for Molecular Analysis of Higher Brain Function, RIKEN Center for Brain Science, Wako, Saitama 351-0198, Japan.

Received: 4 April 2020 Accepted: 19 May 2020

Published online: 27 May 2020

\section{References}

1. Brose K, Tessier-Lavigne M. Slit proteins: key regulators of axon guidance, axonal branching, and cell migration. Curr Opin Neurobiol. 2000;10:95-102.

2. Blockus H, Chedotal A. Slit-Robo Signal Dev. 2016;143:3037-44.

3. Kidd T, Brose K, Mitchell KJ, Fetter RD, Tessier-Lavigne M, Goodman CS, et al. Roundabout controls axon crossing of the CNS midline and defines a novel subfamily of evolutionarily conserved guidance receptors. Cell. 1998;92:205-15.

4. Brose K, Bland KS, Wang KH, Arnott D, Henzel W, Goodman CS, et al. Slit proteins bind Robo receptors and have an evolutionarily conserved role in repulsive axon guidance. Cell. 1999;96:795-806.

5. Sundaresan V, Roberts I, Bateman A, Bankier A, Sheppard M, Hobbs C, et al. The DUTT1 gene, a novel NCAM family member is expressed in developing murine neural tissues and has an unusually broad pattern of expression. Mol Cell Neurosci. 1998;11:29-35.

6. Yuan SS, Cox LA, Dasika GK, Lee EY. Cloning and functional studies of a novel gene aberrantly expressed in RB-deficient embryos. Dev Biol. 1999;207:62-75.

7. Huminiecki L, Gorn M, Suchting S, Poulsom R, Bicknell R. Magic roundabout is a new member of the roundabout receptor family that is endothelial specific and expressed at sites of active angiogenesis. Genomics. 2002;79:547-52.

8. Nguyen-Ba-Charvet KT, Chedotal A. Role of Slit proteins in the vertebrate brain. J Physiol Paris. 2002;96:91-8.

9. Chedotal A. Slits and their receptors. Adv Exp Med Biol. 2007;621:65-80.

10. Plump AS, Erskine L, Sabatier C, Brose K, Epstein CJ, Goodman CS, et al. Slit1 and Slit2 cooperate to prevent premature midline crossing of retinal axons in the mouse visual system. Neuron. 2002;33:219-32.

11. Bagri A, Marin O, Plump AS, Mak J, Pleasure SJ, Rubenstein JL, et al. Slit proteins prevent midline crossing and determine the dorsoventral position of major axonal pathways in the mammalian forebrain. Neuron. 2002;33:233-48.

12. Andrews W, Liapi A, Plachez C, Camurri L, Zhang J, Mori S, et al. Robo1 regulates the development of major axon tracts and interneuron migration in the forebrain. Development. 2006;133:2243-52.

13. Lopez-Bendito G, Flames N, Ma L, Fouquet C, Di Meglio T, Chedotal A, et al. Robo1 and Robo2 cooperate to control the guidance of major axonal tracts in the mammalian forebrain. J Neurosci. 2007;27:3395-407.

14. Koohini Z, Koohini Z, Teimourian S. Slit/Robo signaling pathway in cancer; a new stand point for cancer treatment. Pathol Oncol Res. 2019;25(4):1285-93.

15. Borrell V, Cardenas A, Ciceri G, Galceran J, Flames N, Pla R, et al. Slit/Robo signaling modulates the proliferation of central nervous system progenitors. Neuron. 2012;76:338-52.

16. Amaral DG, Insausti R, Cowan WM. The entorhinal cortex of the monkey: I. Cytoarchitectonic organization. J Comp Neurol. 1987;264:326-55.

17. Gonda Y, Andrews WD, Tabata H, Namba T, Parnavelas JG, Nakajima $\mathrm{K}$, et al. Robo1 regulates the migration and laminar distribution of upper-layer pyramidal neurons of the cerebral cortex. Cereb Cortex. 2013:23:1495-508.

18. Sasaki T, Komatsu Y, Watakabe A, Sawada K, Yamamori T. Prefrontalenriched SLIT1 expression in Old World monkey cortex established during the postnatal development. Cereb Cortex. 2010;20:2496-510.

19. Komatsu Y, Watakabe A, Hashikawa T, Tochitani S, Yamamori T. Retinolbinding protein gene is highly expressed in higher-order association areas of the primate neocortex. Cereb Cortex. 2005;15:96-108.

20. Takaji M, Komatsu Y, Watakabe A, Hashikawa T, Yamamori T. Paraneoplastic antigen-like 5 gene (PNMA5) is preferentially expressed in the association areas in a primate specific manner. Cereb Cortex. 2009;19:2865-79.

21. Yamamori T. Selective gene expression in regions of primate neocortex: implications for cortical specialization. Prog Neurobiol. 2011:94:201-22.

22. Hata K, Mizukami H, Sadakane O, Watakabe A, Ohtsuka M, Takaji M, et al. DNA methylation and methyl-binding proteins control differential gene expression in distinct cortical areas of macaque monkey. J Neurosci. 2013:33:19704-14.

23. Liang F, Hatanaka Y, Saito H, Yamamori T, Hashikawa T. Differential expression of gamma-aminobutyric acid type B receptor-1a and -1b mRNA variants in GABA and non-GABAergic neurons of the rat brain. J Comp Neurol. 2000;416:475-95.

24. Hayashi M, Mistunaga F, Ohira K, Shimizu K. Changes in BDNF-immunoreactive structures in the hippocampal formation of the aged macaque monkey. Brain Res. 2001;918:191-6.

25. Woodhams PL. Laminar and region-specific cell surface markers in the entorhinal cortex and hippocampus. Hippocampus. 1993;3:183-9.

26. Skutella T, Nitsch R. New molecules for hippocampal development. Trends Neurosci. 2001;24:107-13.

27. Suzuki WA, Amaral DG. Where are the perirhinal and parahippocampal cortices? A historical overview of the nomenclature and boundaries applied to the primate medial temporal lobe. Neuroscience. 2003;120:893-906.

28. Witter MP, Doan TP, Jacobsen B, Nilssen ES, Ohara S. Architecture of the entorhinal cortex a review of entorhinal anatomy in rodents with some comparative notes. Front Syst Neurosci. 2017;11:46.

29. Hafting T, Fyhn M, Molden S, Moser MB, Moser El. Microstructure of a spatial map in the entorhinal cortex. Nature. 2005;436:801-6.

30. Moser El, Moser MB, McNaughton BL. Spatial representation in the hippocampal formation: a history. Nat Neurosci. 2017;20:1448-64.

31. Dubois B, Feldman HH, Jacova C, Dekosky ST, Barberger-Gateau P, Cummings J, et al. Research criteria for the diagnosis of Alzheimer's disease: revising the NINCDS-ADRDA criteria. Lancet Neurol. 2007;6:734-46.

32. Hunt MJ, Kopell NJ, Traub RD, Whittington MA. Aberrant network activity in Schizophrenia. Trends Neurosci. 2017:40:371-82.

33. Hagino S, Iseki K, Mori T, Zhang Y, Hikake T, Yokoya S, et al. Slit and glypican-1 mRNAs are coexpressed in the reactive astrocytes of the injured adult brain. Glia. 2003;42:130-8.

34. Murai KK, Pasquale EB. Can Eph receptors stimulate the mind? Neuron. 2002;33:159-62.

35. Nestler EJ. Genes and addiction. Nat Genet. 2000;26:277-81.

36. Bahi A, Dreyer JL. Cocaine-induced expression changes of axon guidance molecules in the adult rat brain. Mol Cell Neurosci. 2005;28:275-91. 
37. Lin L, Lesnick TG, Maraganore DM, Isacson O. Axon guidance and synaptic maintenance: preclinical markers for neurodegenerative disease and therapeutics. Trends Neurosci. 2009;32:142-9.

38. Shen K, Cowan CW. Guidance molecules in synapse formation and plasticity. Cold Spring Harb Perspect Biol. 2010;2:a001842.

39. Duan Y, Wang SH, Song J, Mironova Y, Ming GL, Kolodkin AL, et al. Semaphorin 5A inhibits synaptogenesis in early postnatal- and adult-born hippocampal dentate granule cells. ELife. 2014;3:4390.

40. Holtmaat AJ, Trachtenberg JT, Wilbrecht L, Shepherd GM, Zhang X, Knott $\mathrm{GW}$, et al. Transient and persistent dendritic spines in the neocortex in vivo. Neuron. 2005:45:279-91.
41. Stettler DD, Yamahachi H, Li W, Denk W, Gilbert CD. Axons and synaptic boutons are highly dynamic in adult visual cortex. Neuron. 2006:49:877-87.

42. Sadakane O, Watakabe A, Ohtsuka M, Takaji M, Sasaki T, Kasai M, et al. In vivo two-photon imaging of dendritic spines in marmoset neocortex. ENeuro. 2015. https://doi.org/10.1523/ENEURO.0019-15.2015.

\section{Publisher's Note}

Springer Nature remains neutral with regard to jurisdictional claims in published maps and institutional affiliations.
Ready to submit your research? Choose BMC and benefit from:

- fast, convenient online submission

- thorough peer review by experienced researchers in your field

- rapid publication on acceptance

- support for research data, including large and complex data types

- gold Open Access which fosters wider collaboration and increased citations

- maximum visibility for your research: over $100 \mathrm{M}$ website views per year

At BMC, research is always in progress.

Learn more biomedcentral.com/submissions 